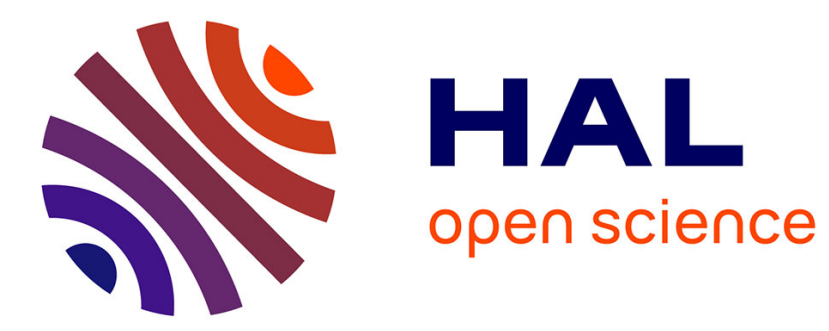

\title{
Embedding simple commutative monoids into simple refinement monoids
}

Friedrich Wehrung

\section{To cite this version:}

Friedrich Wehrung. Embedding simple commutative monoids into simple refinement monoids. Semigroup Forum, 1998, 56 (1), pp.104-129. 10.1007/s00233-002-7008-0 . hal-00004373

\section{HAL Id: hal-00004373 \\ https://hal.science/hal-00004373}

Submitted on 6 Mar 2005

HAL is a multi-disciplinary open access archive for the deposit and dissemination of scientific research documents, whether they are published or not. The documents may come from teaching and research institutions in France or abroad, or from public or private research centers.
L'archive ouverte pluridisciplinaire HAL, est destinée au dépôt et à la diffusion de documents scientifiques de niveau recherche, publiés ou non, émanant des établissements d'enseignement et de recherche français ou étrangers, des laboratoires publics ou privés. 


\title{
Friedrich Wehrung
}

\author{
Communicated by Klaus Keimel
}

\begin{abstract}
Say that a cone is a commutative monoid that is in addition conical, i.e., satisfies $x+y=0 \Rightarrow x=y=0$. We show that cones (resp. simple cones) of many kinds order-embed or even embed unitarily into refinement cones (resp. simple refinement cones) of the same kind, satisfying in addition various divisibility conditions. We do this in particular for all cones, or for all separative cones, or for all cancellative cones (positive cones of partially ordered abelian groups). We also settle both the torsion-free case and the unperforated case. Most of our results extend to arbitrary commutative monoids.
\end{abstract}

\section{$\S 0$. Introduction.}

In [5, Question 30], K. R. Goodearl asks whether every simple partially ordered abelian group can be embedded into a simple Riesz group, and similarly for simple torsion-free (resp. unperforated) partially ordered abelian groups (the condition "simple" has to be added among the hypotheses — indeed, every subgroup of a simple partially ordered abelian group is simple). In this paper we solve positively this question, and many others of the same ilk concerning cones, i.e., conical commutative monoids (not necessarily cancellative). A prototype can be found in P. A. Grillet's [7, Theorem 1], where the author outlines an embedding procedure of every commutative monoid into a refinement monoid; the same result, with a more detailed (and probably independent) proof can be found in H. Dobbertin's [3, Theorem 5.1]; see also [12] for more general results where the preordering is also taken into consideration. The Grillet-Dobbertin embedding result was improved in $[\mathbf{1}]$ by G. M. Bergman, who essentially proved there a version of our Lemma 1.6, which implies in particular that every commutative monoid can be order-embedded into a divisible refinement monoid (in his notes, Bergman uses the notion of "saturated embedding", which is in fact equivalent to the classical notion of unitary embedding [8] and thus equivalent to the definition of unitary embedding adopted in this paper minus cofinality). Using this, Bergman proved also that every simple conical commutative monoid with an infinity (i.e., an element $\infty$ satisfying $(\forall x)(x+\infty=$ $\infty)$ ) embeds into a simple conical refinement monoid with an infinity. Then, using submonoids of products of these with $\mathbb{R}^{+}$, he produced examples of noncancellative directly finite simple refinement monoids, thus solving a problem of Goodearl and O'Meara. Let us emphasize that Bergman's notes [1] contain several other interesting results, and, although our work is related to his, it has been done independently.

Throughout this paper, we will systematically consider additional structural information about both the monoids (separativity, simplicity...) and the

1991 Mathematics subject classification: 06F20, $20 \mathrm{M} 14$.

Key words and phrases: refinement monoids; simple commutative monoids; order-embeddings; unitary embeddings; divisibility. 
embeddings (order-embedding, unitarity, strong unitarity). Thus most of our results will be of the type "every cone of such and such kind admits an embedding of such and such kind into a cone satisfying such and such closure property", and "every cone with such and such closure property is (among other properties) a refinement monoid". Let us be more specific:

- "Every cone of such and such kind...". Some of the classes of cones that we will consider will be the class of all cones, or the class of all cancellative (resp. separative, stably finite, unperforated...) cones.

"... an embedding of such and such kind...". Three sorts of embeddings will be considered: order-embeddings (i.e., monoid homomorphisms that are in addition embeddings for the algebraic preorderings on each side), and the stronger very important notion of unitary embeddings or strong unitary embeddings (Definition 1.2) which will allow us to transfer the embedding results to, e.g., separative cones (it can also been shown, in the language of universal algebra, that any unitary embedding has the "congruence extension property").

- "... such and such closure property". These closure properties will always be maximality properties (similar to algebraic closure) for existence of solutions of finite equation systems (Definition 1.4) in extensions of the monoid under consideration. Their main advantage will be that their models satisfy several properties at once, as refinement, divisibility or quasi-divisibility, etc..

We now summarize briefly each section.

- In section 1, we will prove embedding theorems for cones, without considering simplicity yet. Proposition 1.5 states that cones from a given class embed (sometimes unitarily) into "closed" cones; its proof is in fact a general argument, reminiscent (with a similar proof) of the modeltheoretical fact that every model of a $\forall \exists$ theory embeds into an existentially closed model of that theory (but the latter does not take into account unitary embeddings, nor simplicity). Theorems 1.8 and 1.9 will yield the fact that among other properties, "closed" cones are refinement monoids. This works as well for all cones as, e.g., for cancellative, separative, stably finite, etc., cones. Theorem 1.14 extends this to torsionfreeness or unperforation, but then the following phenomenon happens: either the embedding has very strong properties and we only keep refinement, or the embedding is nothing more than an order-embedding but we have both refinement and divisibility. In a few words,

\section{What is gained for the embedding is lost for the final object, and vice-versa.}

This phenomenon will be present in all our results, and explains their great variety, which forced us to restrict ourselves to a sample. In particular, most of our results extend to the class of all commutative monoids, with very similar proofs, but we chose not to give details about this in order to maintain this paper down to a reasonable size; moreover, the class of commutative monoids which we had in mind often arise from K-theoretical situations, and these are always conical. Proposition 1.16 shows that "closed" cones satisfy the rather mysterious axiom (WSD), which in turn, together with the finite refinement property, guarantees other forms of refinement (as, e.g., the finite interpolation property, or the "hereditary refinement" property (HREF) both considered in [13]).

- Section 2 extends the results of section 1 to simple cones. Proposition 2.5 is the "simple analogue" of Proposition 1.5 (existence of order- 
embeddings or of unitary embeddings into "simply closed" cones), while the "simple analogue" of Theorem 1.9 is Theorem 2.6 ("simply closed" cones are, among other things, refinement cones). It is also in this section that are obtained answers to Goodearl's question for either arbitrary or unperforated partially ordered abelian groups (Corollaries 2.8 and 2.9 ), but not for torsion-free partially ordered abelian groups yet. Finally, Proposition 2.12 shows that "simply closed" cones satisfy (WSD).

- Section 3 is devoted to the remaining torsion-free case, which requires a special treatment because of problems of divisibility - thus the trick of using instead the property of quasi-divisibility (Definition 3.2). We will in fact more generally consider torsion-freeness with respect to a given non-trivial multiplicative subsemigroup $\mathbb{P}$ of $\mathbb{N}$. Then most results of previous sections extend to this context — yielding essentially Theorems 3.5 and 3.9. In particular, the remaining problem about embedding simple torsion-free partially ordered abelian groups into simple torsionfree Riesz groups is solved in Corollary 3.12.

We shall put $\mathbb{N}=\mathbb{Z}^{+} \backslash\{0\}$. If $a_{i}, b_{j}, c_{i j}(i, j<2)$ are elements of some commutative monoid $M$, then we will say that the following array

\begin{tabular}{|l|l|l|}
\cline { 2 - 3 } \multicolumn{1}{c|}{} & $b_{0}$ & $b_{1}$ \\
\hline$a_{0}$ & $c_{00}$ & $c_{01}$ \\
\hline$a_{1}$ & $c_{10}$ & $c_{11}$ \\
\hline
\end{tabular}

is a $(2 \times 2)$ refinement matrix when for all $i<2$, we have $a_{i}=c_{i 0}+c_{i 1}$ and $b_{i}=c_{0 i}+c_{1 i}$. If such a matrix always exists provided that $a_{0}+a_{1}=b_{0}+b_{1}$, then we will say that $M$ is a refinement monoid. If $M$ is a commutative monoid, the algebraic preordering $\leq_{\text {alg }}$ of $M$ is defined by $x \leq_{\text {alg }} y \Leftrightarrow(\exists z)(x+z=y)$ (this terminology is borrowed from the $\mathrm{C}^{*}$-algebraists and we find it more inspired than the term "minimal" used in $[\mathbf{1 1}, \mathbf{1 2}]$, thus we will use it throughout this text). We will often identify a commutative monoid $M$ with its adjunct $\left(M, \leq_{\text {alg }}\right.$ ), emphasized by the denomination "algebraic commutative monoid". The notion of homomorphism is not affected by this adjunction, contrarily to the notion of embedding; thus if $M$ and $N$ are commutative monoids, an order-embedding $f$ from $M$ into $N$ will be an injective homomorphism of monoids which satisfies in addition $x \leq y \Leftrightarrow f(x) \leq f(y)$. If $M$ is a submonoid of a commutative monoid $N$, we will say that $N$ is an extension of $M$ when the inclusion map from $M$ into $N$ is an order-embedding.

An order-unit of a commutative monoid $M$ is an element $u$ of $M$ such that $(\forall x \in M)(\exists n \in \mathbb{N})\left(x \leq_{\text {alg }} n u\right) ; \quad M$ is simple when every $u \in$ $M$ such that $u>0$ (i.e., $\left.u \not \mathbb{X}_{\text {alg }} 0\right)$ is an order-unit of $M$, conical when it satisfies $(\forall x, y)(x+y=0 \Rightarrow x=y=0)$, cancellative when it satisfies $(\forall x, y, z)(x+z=y+z \Rightarrow x=y)$, separative [2, vol. 1] when it satisfies $(\forall x, y)(2 x=x+y=2 y \Rightarrow x=y)$. Note that the latter notion is (strictly) weaker than the notion of separativity used in [12], which was designed there for (positively) preordered monoids.

In addition, if $M$ and $N$ are two commutative monoids and $f: M \rightarrow N$ is a homomorphism of monoids, we will say that $f$ is conical when $f^{-1}\left\{0_{N}\right\}=$ $\left\{0_{M}\right\}$ (note that we write $f^{-1}\{0\}$ instead of $\operatorname{Ker}(f)$, since in monoid-theoretical contexts, the latter denotes in general rather a congruence than a submonoid). Thus if $f$ is one-to-one, then it is conical, but the converse is false.

Furthermore, we will use the canonical transpositions of definitions used for regular rings $[\mathbf{4}, \mathbf{6}]$ to the monoid world: thus, $M$ will be said to be stably 
finite when it satisfies the axiom $(\forall x, y)(x+y=y \Rightarrow x=0)$. We finally refer to [5] for the terminology about partially ordered abelian groups.

\section{$\S 1$. Order-embedding cones.}

In this chapter we will embed algebraic commutative monoids, without considering simplicity yet. In fact, most of our results will concern conical commutative monoids:

Definition 1.1. A cone is a conical commutative monoid; a refinement cone is a cone satisfying the finite refinement property.

We will need some algebraic theory of commutative monoids, in particular the study of amalgamated sums. For this purpose, we will slightly deviate from the original notion of unitary embedding [8, page 232] used in the context of (non-commutative) semigroup amalgams, with the adjunction of a cofinality condition:

Definition 1.2. Let $f: A \rightarrow B$ be a homomorphism of commutative monoids. Then $f$ is unitary when it is one-to-one, has cofinal image (for the algebraic preordering of $B$ ) and it satisfies

$$
\left(\forall a_{0}, a_{1} \in A\right)(\forall b \in B)\left(f\left(a_{0}\right)+b=f\left(a_{1}\right) \Rightarrow b \in f[A]\right) .
$$

We will say that $f$ is strongly unitary when it is unitary and satisfies, for all $m \in \mathbb{N}$, the condition

$$
(\forall b \in B)(m b \in f[A] \Rightarrow b \in f[A]) .
$$

In the case where $A$ is a submonoid of $B$ and $f$ is the inclusion map, we will say that $B$ is a unitary extension (resp. a strong unitary extension) of $A$.

Important examples of unitary and strong unitary embeddings are the following: let $A$ be a cofinal subgroup of a partially ordered abelian group $B$. Then $B^{+}$is a unitary extension of $A^{+}$. If in addition $B / A$ is torsion-free, then $B^{+}$is a strong unitary extension of $A^{+}$; the converse holds provided for example that $A$ is directed.

The proof of the following lemma is straightforward:

\section{Lemma 1.3.}

(a) Every unitary homomorphism of commutative monoids is an order-embedding.

(b) If $f: A \rightarrow B$ and $g: B \rightarrow C$ are unitary (resp. strongly unitary), then $g \circ f$ is unitary (resp. strongly unitary).

(c) If $B$ is a unitary (resp. strong unitary) extension of $A$ and $C$ is a monoid such that $A \subseteq C \subseteq B$, then $C$ is a unitary (resp. strong unitary) extension of $A$.

(d) If $A$ is a commutative monoid, then any directed union of unitary (resp. strong unitary) extensions of $A$ is a unitary (resp. strong unitary) extension of $A$.

If $M$ is a commutative monoid, say that an equation system with parameters from $M$ is a finite set of "equations" each of them of the form

$$
\sum_{i<k} p_{i} \mathbf{x}_{i}+a=\sum_{i<k} q_{i} \mathbf{x}_{i}+b
$$


where $k$ and $p_{i}, q_{i}(i<k)$ are non-negative integers, $a, b$ are elements of $M$ ("parameters") and $\mathbf{x}_{i}(i<k)$ are symbols of variable ("unknowns"). The notion of solution of a given equation system should then be obvious. We will need in this paper three definitions, related to equation systems and unitarity:

Definition 1.4. Let $\mathbf{C}$ be a class of commutative monoids and let $M$ belong to C. Then $M$ is said to be $\mathbf{C}$-closed (resp. C-unitarily closed, $\mathbf{C}$-strongly unitarily closed) when for every equation system $\Sigma$ with parameters from $M$, if $\Sigma$ admits a solution in some extension (resp. some unitary extension, resp. some strong unitary extension) of $M$ belonging to $\mathbf{C}$, then it admits a solution in $M$.

Note the obvious reverse implications

$$
\text { (strongly unitarily closed }) \Leftarrow(\text { unitarily closed }) \Leftarrow(\text { closed }) \text {. }
$$

It would perhaps be preferable, in a more general context, to use the terminology "unitarily algebraically closed", etc., but we choose not to do so for simplicity sake.

If $\mathbf{C}$ is an arbitrary class of commutative monoids, to be "simply $\mathbf{C}$ closed" will be by definition the same as to be $\mathbf{C}^{\prime}$-closed where $\mathbf{C}^{\prime}$ is the class of all simple elements of $\mathbf{C}$. Similar conventions will apply to "simply $\mathbf{C}$-unitarily closed", etc.. A general existence theorem is then the following:

Proposition 1.5. Let $\mathbf{C}$ be a class of commutative monoids closed under unions of chains. Then every element of $\mathbf{C}$ admits an order-embedding (resp. a unitary embedding, a strong unitary embedding) into a $\mathbf{C}$-closed (resp. a Cunitarily closed, $\mathbf{C}$-strongly unitarily closed) element of $\mathbf{C}$.

Proof. Obtained by aping the classical proof of embedding any model of a theory with class of models closed under unions of chains into an existentially closed model: let $M$ be an element of $\mathbf{C}$ and let $\left(\varphi_{\xi}\right)_{\xi<\kappa}$ be an enumeration of all equation systems with parameters from $M$ (where $\kappa$ is an infinite cardinal). Using Lemma 1.3 (d) and the fact that $\mathbf{C}$ is closed under unions of chains, it is not difficult to construct a transfinite chain $\left(M_{\xi}\right)_{\xi<\kappa}$ of elements of $\mathbf{C}$ satisfying the following properties:

(i) If $\xi<\eta<\kappa$, then $M_{\eta}$ is an extension (resp. a unitary extension, a strong unitary extension) of $M_{\xi}$.

(ii) For all $\xi<\kappa$, if $\varphi_{\xi}$ admits a solution in some extension (resp. unitary extension, strong unitary extension) of $M_{\xi}$ in $\mathbf{C}$, then it admits a solution in $M_{\xi+1}$.

Then put $M^{\prime}=\bigcup_{\xi<\kappa} M_{\xi}$. Then $M^{\prime} \in \mathbf{C}$ since $\mathbf{C}$ is closed under unions of chains, and $M^{\prime}$ is an extension (resp. a unitary extension, a strong unitary extension) of $M$. Then define $M^{(n)}\left(n \in \mathbb{Z}^{+}\right)$by $M^{(0)}=M$, and $M^{(n+1)}=\left(M^{(n)}\right)^{\prime}$ for all $n \in \mathbb{Z}^{+}$. Then $\tilde{M}=\bigcup_{n \in \mathbb{Z}^{+}} M^{(n)}$ satisfies the required conditions.

Thus the largest part of this paper will be to investigate the structure of all C-closed (resp. C-unitarily closed, C-strongly unitarily closed) commutative monoids for various classes $\mathbf{C}$. Thus it is natural to start with $\mathbf{C}$ being the class of all commutative monoids.

Lemma 1.6. Let $e: A \rightarrow B$ and $f: A \rightarrow C$ be homomorphisms of commutative monoids. Let $D=B \amalg_{e, f} C$ be the amalgamated sum of $B$ and $C$ along $e$ and $f$ in the category of commutative monoids, so that we have a commutative diagram 




Then the following holds:

(a) If both $B$ and $C$ are conical and if both $e$ and $f$ are conical maps, then $D$ is conical and both $\bar{e}$ and $\bar{f}$ are conical maps (thus $D$ is also the amalgamated sum of $B$ and $C$ along $e$ and $f$ in the category of cones with conical homomorphisms).

(b) If $e$ is unitary, then $\bar{e}$ is also unitary.

(c) If e is strongly unitary, then $\bar{e}$ is also strongly unitary.

In particular, "unitarity and strong unitarity are transferable".

Proof. Let $\rightarrow$ be the binary relation on $B \times C$ consisting exactly on the pairs

$$
(b+e(a), c) \rightarrow(b, f(a)+c) \quad(\text { all } a \in A, b \in B \text { and } c \in C) .
$$

Then $\rightarrow$ is compatible with the addition (i.e., $\xi \rightarrow \eta$ implies $\xi+\zeta \rightarrow \eta+\zeta$ ), thus so is the equivalence relation $\equiv$ on $B \times C$ generated by $\rightarrow$. It follows that $D=B \times C / \equiv$ (up to a natural isomorphism). For all $(b, c) \in B \times C$, denote by $[b, c]$ its natural image in $D$. Then the natural maps $\bar{e}$ and $\bar{f}$ are defined by $\bar{e}(c)=[0, c]$ and $\bar{f}(b)=[b, 0]$.

Let us first settle (a). To prove that $D$ is conical and that $\bar{e}$ and $\bar{f}$ are conical, it suffices, since both $B$ and $C$ are conical, to prove that for all $(b, c) \in B \times C,[b, c]=[0,0]$ implies that $(b, c)=(0,0)$ (the converse being trivial); thus it suffices in fact to prove the conclusion for $(b, c) \rightarrow(0,0)$ and $(0,0) \rightarrow(b, c)$. In the first case, there exists $a \in A$ such that $b=0+e(a)$ and $0=f(a)+c$; by assumption on $C$ and $f$, it follows that $(b, c)=(0,0)$. In the second case, there exists $a \in A$ such that $0=b+e(a)$ and $c=f(a)+0$; by assumption on $B$ and $e$, it follows again that $(b, c)=(0,0)$. The conclusion of (a) follows.

Suppose from now on that $e$ is unitary. Without loss of generality, $A \subseteq B$ and $e$ is the inclusion map from $A$ into $B$, supposed to be unitary.

Since $A$ is cofinal in $B$, for all $(b, c) \in B \times C$, there exists $a \in A$ such that $b \leq a$, thus $[b, c] \leq[a, c]=[0, f(a)+c]=\bar{e}(f(a)+c)$, so that the image of $\bar{e}$ is cofinal in $D$.

Claim. For all $a \in A, b \in B$ and $c, c^{\prime} \in C$, if $(a, c) \equiv\left(b, c^{\prime}\right)$, then $b \in A$ and $f(a)+c=f(b)+c^{\prime}$.

Proof of Claim. It clearly suffices to show that the conclusion holds provided that either $(a, c) \rightarrow\left(b, c^{\prime}\right)$ or $\left(b, c^{\prime}\right) \rightarrow(a, c)$. In the first case, there exists $a^{\prime} \in A$ such that $a=b+a^{\prime}$ and $c^{\prime}=f\left(a^{\prime}\right)+c$; since $e$ is unitary, $b \in A$ and $f(a)+c=f(b)+f\left(a^{\prime}\right)+c=f(b)+c^{\prime}$. In the second case, there exists $a^{\prime} \in A$ such that $b=a+a^{\prime}$ and $c=f\left(a^{\prime}\right)+c^{\prime}$; thus $b \in A$ and $f(a)+c=$ $f(a)+f\left(a^{\prime}\right)+c^{\prime}=f(b)+c^{\prime}$.

From the Claim above, it follows immediately that $\bar{e}$ is one-to-one. Furthermore, if $c_{0}, c_{1} \in C$ and $(b, c) \in B \times C$ such that $\bar{e}\left(c_{0}\right)+[b, c]=\bar{e}\left(c_{1}\right)$, 
i.e., $\left(b, c_{0}+c\right) \equiv\left(0, c_{1}\right)$, then, by the Claim above, $b \in A$, whence $[b, c]=$ $\bar{e}(f(b)+c) \in \bar{e}[C]$, which proves that $\bar{e}$ is unitary; thus (b).

Finally suppose that $e$ is strongly unitary. Let $m \in \mathbb{N}$ and let $[b, c] \in D$ such that $m \cdot[b, c] \in \bar{e}[C]$. This means that there exists $c^{\prime} \in C$ such that $[m b, m c]=\left[0, c^{\prime}\right]$. By the Claim above, $m b \in A$; since $B$ is a strong unitary extension of $A$, it follows that $b \in A$. Therefore, $[b, c]=[0, f(b)+c] \in \bar{e}[C]$. Thus $\bar{e}$ is strongly unitary, and (c) follows.

Now let $R=\left\{\left(x_{0}, x_{1}, x_{2}, x_{3}\right) \in \mathbb{Z}^{4}: x_{0}+x_{3}=x_{1}+x_{2}\right\}$, equipped with the ordering induced by the natural ordering of $\mathbb{Z}^{4}$.

\section{Lemma 1.7.}

(a) $R^{+}$is the submonoid of $\left(\mathbb{Z}^{+}\right)^{4}$ generated by $\alpha_{0}=(1,1,0,0), \alpha_{1}=$ $(0,0,1,1), \beta_{0}=(1,0,1,0)$ and $\beta_{1}=(0,1,0,1)$.

(b) For every commutative monoid $M$ and all elements $a_{i}, b_{i}(i<2)$ of $M$ such that $a_{0}+a_{1}=b_{0}+b_{1}$, there exists a unique homomorphism $f: R^{+} \rightarrow M$ such that for all $i<2, f\left(\alpha_{i}\right)=a_{i}$ and $f\left(\beta_{i}\right)=b_{i}$. If in addition $M$ is conical and $a_{i}, b_{i} \neq 0$ (all $i<2$ ), then $f$ is conical.

Proof. Most of it is well-known - see for example [12, proof of Corollary 2.7]. The last part of (b) is trivial.

We now come to the main theorem of this section (whose full meaning is highlighted by Proposition 1.5):

\section{Theorem 1.8.}

(a) Every strongly unitarily closed cone is a refinement cone.

(b) Let $M$ be a unitarily closed cone. Then $M$ is "normally divisible", i.e., for every $p \in \mathbb{N}$, every finite subset $X$ of $M$ and every element a of $M$, there exists $u \in M$ such that $p u=a$ and for all $x, y \in X, x+a=y+a$ implies $x+u=y+u$.

In the statement of (a), a "strongly unitarily closed cone" is by definition a cone that is strongly unitarily closed relatively to the class of all cones, and similarly for (b).

Proof. We start with (a). Let $a_{i}, b_{i}(i<2)$ be elements of $M$ such that $a_{0}+a_{1}=b_{0}+b_{1}$. Consider the following equation system $\Sigma$ :

$$
\Sigma: \begin{cases}\mathbf{x}_{i 0}+\mathbf{x}_{i 1}=a_{i} & (\text { all } i<2) \\ \mathbf{x}_{0 i}+\mathbf{x}_{1 i}=b_{i} & (\text { all } i<2)\end{cases}
$$

It is trivial that if one of the $a_{i}$ 's or one of the $b_{j}$ 's is zero, then $\Sigma$ admits a solution in $M$. Thus suppose now that all the $a_{i}$ 's and all the $b_{i}$ 's are non zero. By Lemma 1.7, there exists a [unique] conical homomorphism $f: R^{+} \rightarrow M$ such that for all $i<2, f\left(\alpha_{i}\right)=a_{i}$ and $f\left(\beta_{i}\right)=b_{i}$. Let $e$ be the inclusion map from $R^{+}$into $\left(\mathbb{Z}^{+}\right)^{4}$; it is clearly unitary. Moreover, $R$ is the kernel of a homomorphism of abelian groups from $\mathbb{Z}^{4}$ to $\mathbb{Z}$ (namely $\left(x_{0}, x_{1}, x_{2}, x_{3}\right) \mapsto$ $\left.x_{0}+x_{3}-x_{1}-x_{2}\right)$, whence $\mathbb{Z}^{4} / R$ is torsion-free; thus $e$ is strongly unitary. Now let $N=\left(\mathbb{Z}^{+}\right)^{4} \amalg_{e, f} M$; identify $M$ with its natural image into $N$, and let $\bar{f}:\left(\mathbb{Z}^{+}\right)^{4} \rightarrow N$ be the natural homomorphism. By Lemma 1.6, $N$ is conical and also a strong unitary extension of $M$. Furthermore, if $\left(e_{i}\right)_{i<4}$ is the natural basis of $\mathbb{Z}^{4}$, then the following array is a refinement matrix with entries in $N$ :

\begin{tabular}{|c|c|c|}
\cline { 2 - 3 } \multicolumn{1}{c|}{} & $b_{0}$ & $b_{1}$ \\
\hline$a_{0}$ & $\bar{f}\left(e_{0}\right)$ & $\bar{f}\left(e_{1}\right)$ \\
\hline$a_{1}$ & $\bar{f}\left(e_{2}\right)$ & $\bar{f}\left(e_{3}\right)$ \\
\hline
\end{tabular}


Thus $\Sigma$ admits a solution in $N$. Since $N$ is a [conical] strong unitary extension of $M$ and by assumption on $M, \Sigma$ also admits a solution in $M$. Thus $M$ is a refinement cone.

Let us now prove (b). It will also use some unitary extension of $M$, this time constructed directly and not by using some amalgamated sum. The case where $a=0$ is trivial, thus let us suppose from now on that $a \neq 0$. For every real number $r$, denote by $\lceil r\rceil$ the least integer $\geq r$. Then equip $\mathcal{N}=M \times \mathbb{Z}^{+}$with its natural (product) monoid structure, and let $\sim$ the binary relation defined on $\mathcal{N}$ by

$$
(x, m) \sim(y, n) \Longleftrightarrow(m \equiv n \quad(\bmod p) \quad \text { and } \quad x+\lceil m / p\rceil a=y+\lceil n / p\rceil a) .
$$

It is easy to verify that $\sim$ is an equivalence relation on $\mathcal{N}$, which is moreover compatible with the addition of $\mathcal{N}$, i.e., a congruence on $\mathcal{N}$. Put $N=\mathcal{N} / \sim$, and for all $(x, n) \in \mathcal{N}$, denote by $[x, n]$ the equivalence class of $(x, n)$ modulo $\sim$. Since a is non zero and $M$ is conical, it is easy to verify that $N$ is conical. Then let $j: M \rightarrow N, x \mapsto[x, 0]$. It is easy to verify that $j$ is a one-to-one homomorphism from $M$ into $N$. It is unitary: indeed, it is trivially cofinal; further, let $x, y \in M$ and let $(z, n) \in \mathcal{N}$ such that $j(x)+[z, n]=j(y)$; this means that $(x+z, n) \sim(y, 0)$. Thus $n=k p$ for some $k \in \mathbb{Z}^{+}$, and thus $[z, n]=[z+k a, 0]=j(z+k a) \in j[M]$.

Now put $u=[0,1]$. Then $p u=[0, p]=[a, 0]=j(a)$. Furthermore, for all $x$ and $y$ in $M, j(x)+u=j(y)+u$ if and only if $(x, 1) \sim(y, 1)$, i.e., $x+\lceil 1 / p\rceil a=y+\lceil 1 / p\rceil a$, i.e., $x+a=y+a$. Identify $M$ with $j[M]$. Then the following [finite] equation system with unknown $\mathbf{z}$

$$
\left\{\begin{array}{l}
p \mathbf{z}=a \\
x+\mathbf{z}=y+\mathbf{z} \quad(\text { all } x, y \in X \text { such that } x+a=y+a)
\end{array}\right.
$$

admits a solution in $N$, namely $u$. Thus it admits a solution in $M$, which completes the proof of (b).

In particular, every cone embeds strongly unitarily into a refinement cone and every cone embeds unitarily into a normally divisible refinement cone. Now, the interest of having considered unitary embeddings appears in the following theorem, where we only give a sample of the kind of results that similar methods can yield:

Theorem 1.9. Let $\mathbf{C}$ be either the class of all cancellative cones, or the class of all separative cones. Then any $\mathbf{C}$-strongly unitarily closed element of $\mathbf{C}$ is a refinement cone; if in addition it is $\mathbf{C}$-unitarily closed, then it is normally divisible.

Note that it is trivial that both classes above are closed under unions of chains, thus Proposition 1.5 can be used for them.

Proof. In each case, let $M$ be a C-unitarily closed (resp. C-strongly unitarily closed) element of $\mathbf{C}$. By Proposition 1.5 (applied to the class of all cones), $M$ admits a unitary (resp. strong unitary) embedding into a unitarily closed (resp. strongly unitarily closed) cone $N$. There is no reason for $N$ to belong to $\mathbf{C}$, thus the necessity to prove the following

Claim. There exists a least (for the inclusion) congruence $\equiv$ on $N$ such $\hat{N}=N / \equiv$ belongs to $\mathbf{C}$, and then the natural homomorphism $M \rightarrow \hat{N}$ is unitary (resp. strongly unitary). 
Proof of Claim. In the case where $\mathbf{C}$ is the class of all cancellative cones, let $\equiv$ be defined by

$$
x \equiv y \Leftrightarrow(\exists z)(x+z=y+z),
$$

and in the case where $\mathbf{C}$ is the class of all separative cones, let $\equiv$ be defined by

$$
x \equiv y \Leftrightarrow(\exists n \in \mathbb{N})(n x+y=(n+1) x \text { and } x+n y=(n+1) y) .
$$

It is well-known that in both cases, $\equiv$ is the least congruence on $N$ with cancellative (resp. separative) quotient monoid (details about this last fact can be found in [2, vol. 1]).

Thus to conclude the proof of the first part of the statement of the Claim, it suffices to prove that in both cases, $\hat{N}$ is conical. For all $y \in N$, denote by $[y]$ the equivalence class of $y$ modulo $\equiv$. Since $N$ is conical, it suffices to prove that in both cases, for all $y \in N,[y]=[0]$ implies that $y=0$. In the case where $\mathbf{C}$ is the class of all cancellative cones, there exists $x \in N$ such that $y+x=x$; since $M$ is cofinal in $N$, one may take $x \in M$. Since $N$ is a unitary extension of $M$, we get $y \in M$. Since $M$ is cancellative, $y=0$. In the case where $\mathbf{C}$ is the class of all separative cones, there exists $n \in \mathbb{N}$ such that $n y+0=(n+1) y$ and $y+n 0=(n+1) 0$, whence $y=0$. Hence in both cases, $\hat{N}$ is conical.

Now, since $M \in \mathbf{C}$, it is easy to see that $j$ is one-to-one and moreover, since $M$ is cofinal in $N$, the image of $M$ under the natural homomorphism $j: M \rightarrow \hat{N}$ is also cofinal. Moreover, it is not difficult to prove both following statements:

- For all $x_{0}, x_{1} \in M$ and $y \in N$, if $x_{0}+y \equiv x_{1}$, then $y \in M$.

- For all $m \in \mathbb{N}$, all $x \in M$ and all $y \in N, m y \equiv x$ implies that $y \in M$.

(Note that in the case where $\mathbf{C}$ is the class of all cancellative cones, one uses the fact that $M$ is cofinal in $N$ ).

But these two statements (together with the fact that $j$ is one-to-one and cofinal) imply clearly unitarity (resp. strong unitarity) of $j$.

Once this is proved, the conclusion is easily reached: for example for the finite refinement property, if $a_{i}, b_{i}(i<2)$ are elements of $M$ such that $a_{0}+a_{1}=b_{0}+b_{1}$, then, since $N$ is a refinement monoid (this results from Theorem 1.8), the equation system

$$
\begin{cases}\mathbf{x}_{i 0}+\mathbf{x}_{i 1}=a_{i} & (\text { all } i<2) \\ \mathbf{x}_{0 i}+\mathbf{x}_{1 i}=b_{i} & (\text { all } i<2)\end{cases}
$$

admits a solution in $N$, thus also in $\hat{N}$. Since the natural map $M \rightarrow \hat{N}$ is strongly unitary, since both $M$ and $\hat{N}$ belong to $\mathbf{C}$ and by assumption on $M$, the equation system also admits a solution in $M$, which proves that $M$ is a refinement monoid. The proof for normal divisibility runs along the same lines.

Using Proposition 1.5, we immediately get the following

Corollary 1.10. $\quad$ Let $\mathbf{C}$ be either the class of cancellative cones or the class of separative cones. Then every element of $\mathbf{C}$ admits a unitary (resp. strong unitary) embedding into a normally divisible refinement cone (resp. a refinement cone). 
Remark 1.11. Cancellative cones are of course exactly the positive cones of partially ordered abelian groups. Thus, by Corollary 1.10, we get immediately the folklore result that every directed partially ordered abelian group $A$ embeds into a Riesz group $B$ (a Riesz group is a directed interpolation group). While unitarity gives no additional information on the embedding in the case of groups, it allows to have $B$ divisible (and even with divisible positive cone); on the other hand, strong unitarity yields $B / A$ torsion-free (but one may no longer be able to maintain divisibility of $B$ ), in which case $B$ inherits whatever torsion-freeness $A$ enjoys. In particular, every directed torsion-free partially ordered abelian group $A$ embeds into a torsion-free Riesz group $B$ such that $B / A$ is torsion-free; see also Corollary 1.15.

In fact, it is not difficult to prove that every (not necessarily directed) partially ordered abelian group $A$ embeds cofinally into an interpolation group $B$ whose underlying group is of the form $A \oplus F$ where $F$ is free abelian (on $\left|A^{+}\right|$ generators), thus yielding both results above; see also Remark 3.13.

As an extension of Corollary 1.10 to further classes of cones, note for example the following

Corollary 1.12. Every stably finite separative cone embeds unitarily (resp. strongly unitarily) into a normally divisible stably finite separative refinement cone (resp. a stably finite separative refinement cone).

Proof. Note that if $N$ is a unitary extension of $M$ and $M$ is stably finite, then $N$ is also stably finite. Then apply Corollary 1.10 .

Nevertheless, as we shall now see, it is not always possible to preserve unitarity in all these embedding theorems. Theorem 1.14 below gives a sample of this kind of situation.

Definition 1.13. Let $\mathbb{P}$ be a multiplicative subsemigroup of $\mathbb{N}$. Say that a commutative monoid is $\mathbb{P}$-torsion-free (resp. $\mathbb{P}$-unperforated) when for all $p \in \mathbb{P}$, it satisfies the axiom $(\forall x, y)(p x=p y \Rightarrow x=y)$ (resp. $(\forall x, y)(p x \leq p y \Rightarrow x \leq$ $y)$ ). Denote by $\mathbf{C}_{\mathbb{P}}\left(\right.$ resp. $\left.\mathbf{C}_{\mathbb{P}}^{\prime}\right)$ the class of all $\mathbb{P}$-torsion-free (resp. $\mathbb{P}$-torsion-free and $\mathbb{P}$-unperforated) cones.

\section{Theorem 1.14.}

(a) Every $\mathbf{C}_{\mathbb{P}}$-strongly unitarily closed cone is a refinement cone.

(b) Every $\mathbf{C}_{\mathbb{P}}^{\prime}$-closed cone is a normally divisible refinement cone.

Proof. (a) Let $M$ be a $\mathbf{C}_{\mathbb{P}}$-strongly unitarily closed cone. By Proposition 1.5, $M$ embeds strongly unitarily into a strongly unitarily closed cone $N$. By Theorem 1.8, $N$ is a refinement cone. Let $\equiv$ be the congruence on $N$ defined by

$$
x \equiv y \Leftrightarrow(\exists p \in \mathbb{P})(p x=p y) .
$$

It is trivial that $N / \equiv$ is conical. By definition, $N / \equiv$ is $\mathbb{P}$-torsion-free. It is also easy to see that the natural homomorphism $M \rightarrow N / \equiv$ is strongly unitary.

Now the argument used (for the refinement) at the end of the proof of Theorem 1.9 allows us easily to conclude that $M$ is a refinement cone.

Let us now prove (b). Thus let $M$ be a $\mathbf{C}_{\mathbb{P}}^{\prime}$-closed cone. By Proposition 1.5, $M$ order-embeds into a closed cone $N$. By Theorem 1.8, $N$ is a normally divisible refinement cone. As above, let $\equiv$ be the congruence on $N$ defined by

$$
x \equiv y \Leftrightarrow(\exists p \in \mathbb{P})(p x=p y) .
$$


It is again trivial that $N / \equiv$ is conical. By definition, $N / \equiv$ is $\mathbb{P}$-torsionfree; by using the fact that $N$ is divisible, one can also see easily that $N / \equiv$ is $\mathbb{P}$-unperforated. Finally, one checks easily that the natural homomorphism $M \rightarrow N / \equiv$ is an order-embedding. From then on, the usual argument shows that $M$ is a normally divisible refinement monoid.

\section{Corollary 1.15.}

(a) Every $\mathbb{P}$-torsion-free cone embeds strongly unitarily into a $\mathbb{P}$-torsion-free refinement cone.

(b) Every directed $\mathbb{P}$-torsion-free partially ordered abelian group $A$ embeds cofinally into a $\mathbb{P}$-torsion-free Riesz group $B$ such that $B / A$ is torsionfree.

(c) Every $\mathbb{P}$-torsion-free $\mathbb{P}$ - unperforated cone order-embeds into a $\mathbb{P}$-torsionfree $\mathbb{P}$-unperforated normally divisible refinement cone.

(d) Every directed $\mathbb{P}$-unperforated partially ordered abelian group $A$ embeds cofinally into a $\mathbb{P}$-unperforated Riesz group $B$ such that $B / A$ is torsionfree.

Proof. (a), (c) result immediately from Proposition 1.5 and Theorem 1.14, while (b) results immediately from Remark 1.11. Let us see now (d); thus let $\mathbf{C}$ be the class of all $\mathbb{P}$-unperforated cancellative cones and let $M$ be a $\mathbf{C}$-strongly unitarily closed cone; thus $M=A^{+}$for some directed partially ordered abelian group $A$. By (b) above, $A$ embeds cofinally into a $\mathbb{P}$-torsion free Riesz group $B$ such that $B / A$ is torsion-free. Now define a subset $P$ of $B$ by

$$
P=\{x \in B:(\exists p \in \mathbb{P})(p x \geq 0)\} .
$$

Using the fact that $B$ is $\mathbb{P}$-torsion-free, one sees easily that $P$ is the positive cone of a $\mathbb{P}$-unperforated partially ordered abelian group $B^{\prime}$ of underlying group $B$; in particular, $P \in \mathbf{C}$. Since $A$ is $\mathbb{P}$-unperforated, the inclusion map from $A$ into $B^{\prime}$ is an order-embedding; since $P$ contains $B^{+}$, it is still cofinal; thus, since $B / A$ is torsion-free, the inclusion map from $M$ into $P$ is strongly unitary. If $a_{i}, b_{i}(i<2)$ are elements of $M$ such that $a_{0}+a_{1}=b_{0}+b_{1}$, then, since $B^{+}$ is a refinement cone, the equation system

$$
\Sigma: \begin{cases}\mathbf{x}_{i 0}+\mathbf{x}_{i 1}=a_{i} & (\text { all } i<2) \\ \mathbf{x}_{0 i}+\mathbf{x}_{1 i}=b_{i} & (\text { all } i<2)\end{cases}
$$

admits a solution in $B^{+}$, thus a fortiori in $P$. By assumption on $M, \Sigma$ admits a solution in $M$; whence $M$ is a refinement cone. We conclude again by Proposition 1.5.

Note that one cannot improve "refinement cone" into "divisible refinement cone" in (a) above (because every divisible torsion-free commutative monoid is unperforated while there are torsion-free perforated cones); however, this drawback will be overcome in section 3 where we will introduce quasi-divisibility. Note also that one cannot improve "order-embeds" into "embeds unitarily" in (c) above: for example, $M=\mathbb{Z}^{+} \cup\{\infty\}$ does not embed unitarily into any divisible, torsion-free unperforated commutative monoid (because in such an extension, multiplication by positive rational numbers would be defined, and then $(1 / 2)+\infty=\infty$ while $1 / 2 \notin M)$.

We shall finally discuss briefly another property, the axiom (WSD) ("Weak Sum Decomposition"), slightly weaker than the axiom (SD) considered in $[\mathbf{1 3}]$ :

$$
\begin{aligned}
& \left(\forall a_{0}, a_{1}, b, c\right)\left[a_{0}+a_{1}+c=b+c \Rightarrow\right. \\
& \left.\left(\exists x_{0}, x_{1}\right)\left(a_{0}+c=x_{0}+c \text { and } a_{1}+c=x_{1}+c \text { and } b=x_{0}+x_{1}\right)\right] .
\end{aligned}
$$


It is easy to verify that every cone satisfying (WSD) is antisymmetric (i.e., it satisfies $(\forall x, y)((x \leq y$ and $y \leq x) \Rightarrow x=y))$, but it should be noted that (WSD) is not a consequence of the finite refinement property plus antisymmetry. A simple counterexample for this is the monoid $\Lambda\left(\mathbb{Q}^{+}\right)$of all nonempty lower subsets of $\mathbb{Q}^{+}$: for any non-negative real number $r$, identify $r$ with the interval $[0, r] \cap \mathbb{Q}^{+}$, and put $r^{-}=[0, r) \cap \mathbb{Q}^{+}$(so that for irrational $r$, we have $r=r^{-}$); then, let $\alpha$ be any irrational number such that $0<\alpha<1$, and take $\mathfrak{a}_{0}=\alpha$, $\mathfrak{a}_{1}=1-\alpha, \mathfrak{b}=1$ and $\mathfrak{c}=1^{-} ;$then $\mathfrak{a}_{0}, \mathfrak{a}_{1}, \mathfrak{b}, \mathfrak{c}$ witness failure of (WSD) in $\Lambda\left(\mathbb{Q}^{+}\right)$; see also the more general [13, Theorem 2.21]. Another source of counterexamples (that refinement does not imply (WSD)) comes from the fact that refinement plus (WSD) implies that the maximal cancellative quotient has refinement (it implies in fact $(\mathrm{HREF})$ as defined in $[\mathbf{1 3}]$, but it is not equivalent to (HREF); for example, $\boldsymbol{\Lambda}\left(\mathbb{Q}^{+}\right)$satisfies (HREF) [13, Theorem 2.11]): so for J. Moncasi's example of a regular ring $R$ such that $K_{0}(R)$ is not a Riesz group, the cone of isomorphism classes of finitely generated projective right $R$-modules satisfies refinement but not (WSD); similarly, if $A$ and $B$ are Riesz groups such that $A \otimes B$ is not an interpolation group [14, Example 1.4], the tensor product of $A^{+}$and $B^{+}$as commutative monoids satisfies refinement [14, Theorem 2.9], but not (WSD). Our next proposition will produce cones satisfying (WSD).

Proposition 1.16. Let $\mathbf{C}$ be the class of all antisymmetric cones. Then every C-closed cone satisfies (WSD).

Proof. Let $M$ be a $\mathbf{C}$-closed cone. Let $a_{0}, a_{1}, b$ and $c$ in $M$ such that $a_{0}+a_{1}+c=b+c$. Consider the following equation system with unknowns $\mathbf{x}_{0}$ and $\mathbf{x}_{1}$ :

$$
\Sigma:\left\{\begin{array}{l}
\mathbf{x}_{0}+c=a_{0}+c \\
\mathbf{x}_{1}+c=a_{1}+c ; \\
\mathbf{x}_{0}+\mathbf{x}_{1}=b .
\end{array}\right.
$$

We shall construct an extension $N$ of $M$ in $\mathbf{C}$ where $\Sigma$ admits a solution.

If $a_{0}=0$ then $\left(x_{0}=0 ; x_{1}=b\right)$ is a solution of $\Sigma$ in $M$. Similarly for $a_{1}=0$. If $b=0$, then, since $M$ is antisymmetric, $a_{0}+c=a_{1}+c=c$, thus $x_{0}=x_{1}=0$ is a solution of $\Sigma$ in $M$.

So now, suppose that $a_{0}, a_{1}$ and $b$ are non zero. Let $F=\mathbb{Z}^{+} \times \mathbb{Z}^{+}$and let $f: F \rightarrow M$ be the unique homomorphism of monoids sending $e_{0}=(1,0)$ on $a_{0}$ and $e_{1}=(0,1)$ on $a_{1}$. Let $\rightarrow$ the binary relation ("rewriting rule") on $\mathcal{N}=M \times F$ consisting exactly on all pairs of the form

$$
\begin{gathered}
(x, r) \rightarrow(x, r), \\
\left(x, e_{i}+r\right) \rightarrow\left(x+a_{i}, r\right) \quad(\text { for } i<2) \quad \text { if } x \geq c, \\
\left(x, e_{0}+e_{1}+r\right) \rightarrow(x+b, r) .
\end{gathered}
$$

It is immediate to verify that $\rightarrow$ is compatible with the addition of $\mathcal{N}$ (i.e., $\xi \rightarrow \eta$ implies $\xi+\zeta \rightarrow \eta+\zeta$ ).

Claim. The relation $\rightarrow$ is confluent, i.e., for all $\xi, \eta_{0}, \eta_{1}$ in $\mathcal{N}$ such that $\xi \rightarrow \eta_{i}$ (all $i<2$ ), there exists $\zeta \in \mathcal{N}$ such that $\eta_{i} \rightarrow \zeta$ (all $i<2$ ).

Proof of Claim. There are essentially two cases that are not completely trivial to consider:

Case 1. $\xi=(x, r)$ with $r=e_{0}+r_{0}=e_{1}+r_{1}$ and $x \geq c$, and $\eta_{i}=\left(x+a_{i}, r_{i}\right)$ (all $i<2)$. Then there exists $r^{\prime} \in F$ such that $r_{i}=e_{1-i}+r^{\prime}($ all $i<2)$. Take $\zeta=\left(x+a_{0}+a_{1}, r^{\prime}\right)$.

Case 2. $\xi=\left(x, e_{0}+e_{1}+r\right)$ with $x \geq c, \eta_{0}=\left(x+a_{0}, e_{1}+r\right)$ and $\eta_{1}=(x+b, r)$. Then since $x \geq c$, we have $x+a_{0}+a_{1}=x+b$, and then it is easy to verify that one can take $\zeta=\eta_{1}=(x+b, r)$. 
Now let $\rightarrow^{*}$ be the transitive closure of $\rightarrow$. Since $\rightarrow$ is reflexive, $\rightarrow^{*}$ is reflexive; since $\rightarrow$ is compatible with the addition, $\rightarrow^{*}$ is also compatible with the addition, and since $\rightarrow$ is confluent, $\rightarrow^{*}$ is also confluent. Thus the binary relation $\equiv$ defined on $\mathcal{N}$ by

$$
\xi \equiv \eta \Leftrightarrow(\exists \zeta)\left(\xi \rightarrow^{*} \zeta \text { and } \eta \rightarrow^{*} \zeta\right)
$$

is a congruence on $\mathcal{N}$. Let $N=\mathcal{N} / \equiv$, and for all $(x, r) \in \mathcal{N}$, denote by $[x, r]$ its equivalence class modulo $\equiv$. To prove that $N$ is conical, it suffices to prove that for all $(x, r) \in M \times F,[x, r]=[0,0]$ implies that $(x, r)=(0,0)$. Thus suppose $[x, r]=[0,0]$. By definition, there exists $(y, s) \in M \times F$ such that $(x, r) \rightarrow^{*}(y, s)$ and $(0,0) \rightarrow^{*}(y, s)$. The second condition easily implies that $(y, s)=(0,0)$, whence $(x, r) \rightarrow^{*}(0,0)$. Since $\rightarrow$ increases the first coordinate, $x \leq 0$, thus, by conicality of $M, x=0$, so that $(0, r) \rightarrow^{*}(0,0)$. But since $a_{0}, a_{1}$ and $b$ are non zero and $M$ is conical, this is possible only when $r=0$; whence $N$ is conical.

Further, let $j: M \rightarrow N, x \mapsto[x, 0]$. It is immediate that $j$ is a monoid homomorphism. If $x$ and $y$ are two elements of $M$ such that $j(x)=j(y)$, then there exists $(z, r) \in \mathcal{N}$ such that $(x, 0) \rightarrow^{*}(z, r)$ and $(y, 0) \rightarrow^{*}(z, r)$. Since $\rightarrow$ strictly decreases the second coordinate (except in the cases where it is equality), we necessarily have $r=0$ and $z=x=y$. Now if we just suppose that $j(x) \leq j(y)$, then there exists $(z, r) \in \mathcal{N}$ such that $(x+z, r) \equiv(y, 0)$, thus there exists $\left(y^{\prime}, r^{\prime}\right) \in \mathcal{N}$ such that $(x+z, r) \rightarrow^{*}\left(y^{\prime}, r^{\prime}\right)$ and $(y, 0) \rightarrow^{*}\left(y^{\prime}, r^{\prime}\right)$. Thus, as before, $r^{\prime}=0$ and $y^{\prime}=y$; since $x+z \leq y^{\prime}$, we deduce $x \leq y$. Therefore, $j$ is an order-embedding from $M$ into $N$.

Further, put $x_{i}=\left[0, e_{i}\right]$ for all $i<2$. Then $x_{i}+j(c)=\left[c, e_{i}\right]=$ $\left[c+a_{i}, 0\right]=j\left(a_{i}\right)+j(c)$. Furthermore, $x_{0}+x_{1}=\left[0, e_{0}+e_{1}\right]=[b, 0]=j(b)$. Thus, the image of $\Sigma$ under $j$ admits a solution in $N$.

Finally, let $N^{\prime}$ be the maximal antisymmetric quotient of $N$ : that is, $N^{\prime}=N / \equiv$ where $\equiv$ is defined by $x \equiv y \Leftrightarrow(x \leq y$ and $y \leq x)$ (it is obviously a congruence on $N$ ), let $\pi: N \rightarrow N^{\prime}$ be the natural projection. Then $\pi \circ j$ is an order-embedding from $M$ into $N^{\prime}$, and the image of $\Sigma$ under $\pi \circ j$ admits a solution in $N^{\prime}$. Since $M$ is $\mathbf{C}$-closed, $\Sigma$ also admits a solution in $M$, which concludes the proof.

Corollary 1.17. Every antisymmetric cone order-embeds into a normally divisible refinement cone satisfying (WSD).

Note that it is easy to verify that every normally divisible refinement monoid satisfying (WSD) satisfies in fact the axiom (SD) of [13]: thus it is a refinement algebra in the sense of $[\mathbf{1 3}]$.

\section{$\S 2$. Order-embedding simple cones.}

In this section we will try to extend the results of previous section to simple cones. A drawback to this is that we will need to use divisibility in several cases, even for the mere proof of the finite refinement property; thus this section will contain no result concerning strongly unitary embeddings. We will return back to the latter in section 3, to solve the remaining questions. We shall make use of the following lemma [11, Lemma 1.9]: 
Lemma 2.1. Let $n \in \mathbb{N}$, let $M$ be a refinement monoid and let $a, b$ and $c$ be elements of $M$ such that $a+b=n c$. Then there exist elements $c_{k}(0 \leq k \leq n)$ of $M$ such that

$$
a=\sum_{k \leq n} k c_{k}, \quad b=\sum_{k \leq n}(n-k) c_{k} \quad \text { and } \quad c=\sum_{k \leq n} c_{k} .
$$

For all elements $a$ and $b$ of an algebraic commutative monoid $M$, we will write $a \propto b$ when there exists $n \in \mathbb{N}$ such that $a \leq n b$, and $a \asymp b$ when $a \propto b$ and $b \propto a$. Furthermore, for all $a \in M$, put $M(a)=\{x \in M: x \asymp a\} \cup\{0\}$. A subset $X$ of $M$ is $\asymp-t r i v i a l$ when $(\forall x, y \in X)(x \asymp y)$. Say that a refinement matrix is $\asymp$-trivial when the set of all its entries is $\asymp$-trivial.

Lemma 2.2. Let $M$ be a refinement monoid. Then every single $\asymp$-equivalence class of $M$ is downward directed.

Proof. It suffices to prove that if $a$ and $b$ are two elements of $M$ such that $a \asymp b$, then there exists $c \asymp a$ such that $c \leq a$ and $c \leq b$. By assumption there exists $n \in \mathbb{N}$ such that $a \leq n b$. By Lemma 2.1, there are elements $c_{k}$ $(0 \leq k \leq n)$ such that $a=\sum_{k \leq n} k c_{k}$ and $b=\sum_{k \leq n} c_{k}$. Put $c=\sum_{k=1}^{n} c_{k}$. Then $c \leq a$ and $c \leq b$; thus $c \propto \bar{a}$, but $a \leq n c$, thus $a \asymp c$.

We now come to the main lemma of this section:

Lemma 2.3. Let $M$ be a cone and let $a \in M$. Then $M(a)$ is a simple cone. If in addition $M$ is a normally divisible refinement cone, then $M(a)$ is a simple normally divisible refinement cone.

Note that in fact, in order to get refinement in $M(a)$, the hypothesis of the second paragraph of Lemma 2.3 can be weakened into " $M$ is a refinement cone satisfying the axiom

$$
(\forall a, b, c)(a+c=b+c \Rightarrow(\exists x)(2 x=c \text { and } a+x=b+x)) "
$$

as the proof will show it.

Proof. It is obvious that $M(a)$ is a submonoid of $M$. Now let $x$ and $y$ be two non zero elements of $M(a)$. Since $x \asymp y$, there are $n \in \mathbb{N}$ and $z \in M$ such that $x+z=n y$. Therefore, $y+z \in M(a)$ and $x+(y+z)=(n+1) y$, so that $M(a)$ satisfies $x \propto y$; therefore, $y$ is an order-unit of $M(a)$; whence $M(a)$ is simple.

From now on suppose that $M$ is a normally divisible refinement monoid. Let $a_{0}, a_{1}, b_{0}$ and $b_{1}$ be elements of $M(a)$ such that $a_{0}+a_{1}=b_{0}+b_{1}$; we must find a refinement of this equality in $M(a)$. If one of the $a_{i}$ 's or one of the $b_{i}$ 's is equal to 0 , then the problem obviously admits a solution, thus suppose that none of the $a_{i}, b_{i}$ 's is equal to zero. Put $X=\left\{a_{0}, a_{1}, b_{0}, b_{1}\right\}$. By Lemma 2.2, there exists $c \asymp a$ such that $c \leq X$. Thus there are elements $a_{i}^{\prime}, b_{i}^{\prime}(i<2)$ of $M$ such that $a_{i}=c+a_{i}^{\prime}$ and $b_{i}=c+b_{i}^{\prime}($ all $i<2)$, whence $a_{0}^{\prime}+a_{1}^{\prime}+2 c=b_{0}^{\prime}+b_{1}^{\prime}+2 c$. Now, two successive applications of normal divisibility of $M$ yield two elements $u_{0}$ and $u_{1}$ of $M$ such that $2 u_{0}=2 u_{1}=c$ and $a_{0}^{\prime}+a_{1}^{\prime}+u_{0}+u_{1}=b_{0}^{\prime}+b_{1}^{\prime}+u_{0}+u_{1}$. Thus $u_{0} \asymp u_{1}$, thus, since $M$ is a refinement monoid and by Lemma 2.2 , there exists $u \in M$ such that $u \leq\left\{u_{0}, u_{1}\right\}$ and $u \asymp\left\{u_{0}, u_{1}\right\}$. Thus there are elements $u_{0}^{\prime}$ and $u_{1}^{\prime}$ of $M$ such that $u_{i}=u+u_{i}^{\prime}($ all $i<2)$. Then one more application 
of normal divisibility yields an element $v$ of $M$ such that $2 v=u$. Now we have the following refinement matrix

\begin{tabular}{|c|c|c|}
\cline { 2 - 3 } \multicolumn{1}{c|}{} & $u_{0}$ & $u_{1}$ \\
\hline$u_{0}$ & $v+u_{0}^{\prime}$ & $v$ \\
\hline$u_{1}$ & $v$ & $v+u_{1}^{\prime}$ \\
\hline
\end{tabular}

and it is $\asymp$-trivial (because $v \asymp u \asymp u_{0} \asymp u_{1}$ ). Furthermore, since $M$ is a refinement monoid, there exists a refinement matrix with entries in $M$ of the following form:

\begin{tabular}{|c|c|c|}
\cline { 2 - 3 } \multicolumn{1}{c|}{} & $b_{0}^{\prime}+u_{0}$ & $b_{1}^{\prime}+u_{1}$ \\
\hline$a_{0}^{\prime}+u_{0}$ & $c_{00}^{\prime}$ & $c_{01}^{\prime}$ \\
\hline$a_{1}^{\prime}+u_{1}$ & $c_{10}^{\prime}$ & $c_{11}^{\prime}$ \\
\hline
\end{tabular}

Therefore, we have the following refinement matrix in $M$ :

\begin{tabular}{|c|c|c|}
\cline { 2 - 3 } \multicolumn{1}{c|}{} & $b_{0}$ & $b_{1}$ \\
\hline$a_{0}$ & $c_{00}^{\prime}+v+u_{0}^{\prime}$ & $c_{01}^{\prime}+v$ \\
\hline$a_{1}$ & $c_{10}^{\prime}+v$ & $c_{11}^{\prime}+v+u_{1}^{\prime}$ \\
\hline
\end{tabular}

and it is $\asymp$-trivial (all its entries are $\geq v$ ); thus all its entries belong to $M(a)$.

Let us finally verify normal divisibility. Thus let $p \in \mathbb{N}$ and let $b \in M(a)$ and $X \subseteq M(a)$ be a finite subset. Put $Y=\{(x, y) \in X \times X: x+b=y+b\}$. Since $M$ is normally divisible, there exists $c \in M$ such that $p c=b$ and $(\forall(x, y) \in Y)(x+c=y+c)$. But $c \asymp b$, thus $c \in M(a)$, and we are done.

Remark 2.4. It is not difficult to prove that for every simple atomless refinement cone $M$, the semigroup $M^{>0}=\{x \in M: x>0\}$ satisfies the finite refinement property (this fails of course for $M=\mathbb{Z}^{+}$, which is atomic); in fact, the proof works for atomless refinement cones $M$ that are in addition prime, i.e., $M^{>0}$ is downward directed. A proof of this can be found in Bergman's notes [1]. This can also be considered as a vindication of the result of Lemma 2.3.

Before going on, let us rephrase for convenience the version of Proposition 1.5 for simple commutative monoids:

Proposition 2.5. Let $\mathbf{C}$ be a class of commutative monoids closed under unions of chains. Then every simple commutative monoid of $\mathbf{C}$ admits an orderembedding (resp. a unitary embedding, a strong unitary embedding) into a simply $\mathbf{C}$-closed (resp. a simply $\mathbf{C}$-unitarily closed, a simply $\mathbf{C}$-strongly unitarily closed) element of $\mathbf{C}$.

Proof. Just apply Proposition 1.5 to the class of all simple elements of $\mathbf{C}$ (the point is that the union of any chain of simple commutative monoids is a simple commutative monoid).

Note that the class of all cones (resp. of all cancellative, of all separative cones) is closed under unions of chains, thus satisfies the hypothesis of Proposition 2.5 .

Now we can prove our main embedding theorem for simple refinement cones: 
Theorem 2.6. $\quad$ Let $\mathbf{C}$ be the class of all cones (resp. cancellative, resp. separative cones). Then every simply $\mathbf{C}$-unitarily closed element of $\mathbf{C}$ is a normally divisible refinement cone.

Proof. Let $M$ be a simply $\mathbf{C}$-unitarily closed element of $\mathbf{C}$. By Proposition 1.5, $M$ admits a unitary embedding into a $\mathbf{C}$-unitarily closed element $N$ of $\mathbf{C}$. By Theorems 1.8 and 1.9, $N$ is a normally divisible refinement cone.

Let us first prove that $M$ is a refinement monoid. Thus let $a_{i}, b_{i}(i<2)$ be elements of $M$ such that $a_{0}+a_{1}=b_{0}+b_{1}$. As usual, let $\Sigma$ be the following equation system with unknowns $\mathbf{x}_{i j}(i, j<2)$ :

$$
\Sigma: \begin{cases}\mathbf{x}_{i 0}+\mathbf{x}_{i 1}=a_{i} & (\text { all } i<2) \\ \mathbf{x}_{0 i}+\mathbf{x}_{1 i}=b_{i} & (\text { all } i<2)\end{cases}
$$

If one of the $a_{i}, b_{i}$ 's is equal to zero, then it is trivial that $\Sigma$ admits a solution in $M$.

So now, suppose that neither of the $a_{i}, b_{i}$ 's is equal to zero. Then, since $M$ is simple, all four of them are order-units, thus $a_{0} \asymp a_{1} \asymp b_{0} \asymp b_{1}$. By Lemma 2.3, $\Sigma$ admits a solution in $N\left(a_{0}\right)$. Since $M$ is simple, we have $M \subseteq N\left(a_{0}\right)$; since $N$ is a unitary extension of $M$, so is $N\left(a_{0}\right)$. Since $N\left(a_{0}\right) \in \overline{\mathbf{C}}$ and $M$ is simply $\mathbf{C}$-unitarily closed, $\Sigma$ also admits a solution in $M$. Thus $M$ is a refinement monoid.

Let now $X$ be a finite subset of $M$ and let $a \in M$. Put $Y=\{(x, y) \in$ $X \times X: x+a=y+a\}$ and consider the following equation system with unknown $\mathbf{Z}$

$$
\Sigma^{\prime}:\left\{\begin{array}{l}
p \mathbf{z}=a \\
x+\mathbf{z}=y+\mathbf{z} \quad(\text { all }(x, y) \in Y)
\end{array}\right.
$$

If $a=0$, then it is obvious that $\Sigma^{\prime}$ admits a solution in $M$, thus suppose that $a \neq 0$. Thus all parameters of $\Sigma^{\prime}$ belong to $N(a)$, thus, since $N(a)$ is normally divisible (by Lemma 2.3), $\Sigma^{\prime}$ admits a solution in $N(a)$. But as for refinement, $N(a)$ is a unitary extension of $M$ which belongs to $\mathbf{C}$, whence $\Sigma^{\prime}$ admits a solution in $M$. Hence $M$ is normally divisible.

Corollary 2.7. $\quad$ Let $\mathbf{C}$ be the class of all cones (resp. cancellative, resp. separative cones). Then every simple element of $\mathbf{C}$ embeds unitarily into a simple normally divisible refinement cone belonging to $\mathbf{C}$.

By applying again Remark 1.11, one can easily extend this result to other classes of cones. For example, by taking for $\mathbf{C}$ the class of all cancellative cones, one obtains easily the following result:

Corollary 2.8. $\quad$ Every simple partially ordered abelian group embeds into a simple Riesz group with divisible positive cone.

(Note also that an [order-] embedding of simple partially ordered abelian groups is necessarily cofinal).

In fact, it is not difficult to extend this result to the class of unperforated partially ordered abelian groups:

Corollary 2.9. $\quad$ Every simple unperforated partially ordered abelian group embeds into a simple divisible dimension group.

Proof. Denote by $\mathbf{C}$ the class of all cancellative cones (i.e., the class of all positive cones of partially ordered abelian groups), and by $\mathbf{C}^{\prime}$ the class of all 
unperforated elements of C. By Proposition 2.5, it suffices to prove that every simply $\mathbf{C}^{\prime}$-closed cone $M$ is a divisible refinement cone. By Corollary 2.8, $M$ order-embeds into the positive cone $N$ of some simple Riesz group $H$, with $N$ divisible. Now define a convex subgroup $I$ of $H$ by

$$
I=\{x \in H:(\exists m \in \mathbb{N})(m x=0)\},
$$

and let $\hat{N}$ be the positive cone of $H / I$. Then it is obvious, using torsion-freeness of $M$, that the natural homomorphism $j: M \rightarrow \hat{N}$ is one-to-one. It is also easy to see, using unperforation, that $j$ is an order-embedding. Furthermore, using the fact that $N$ is divisible, it is not difficult to infer that $\hat{N}$ is unperforated (thus belongs to $\mathbf{C}^{\prime}$ ) and divisible.

Now let $a \in M$ and let $m \in \mathbb{N}$. Then, since $N$ is divisible, the equation $m \mathbf{x}=a$ admits a solution in $N$, thus in $\hat{N}$; thus, by assumption on $M$, the equation also admits a solution in $M$. Therefore, $M$ is divisible. The proof for refinement is similar (see also the proof of Theorem 1.14 (b)).

A similar application of Corollary 2.7 yields also, with a proof similar to the one of Theorem 1.14 (b), the following result (recall that $\mathbb{P}$ is a given multiplicative subsemigroup of $\mathbb{N}$ ):

Corollary 2.10. $\quad$ Every simple $\mathbb{P}$-torsion-free $\mathbb{P}$-unperforated cone orderembeds into a normally divisible simple $\mathbb{P}$-torsion-free $\mathbb{P}$-unperforated refinement cone.

Remark 2.11. For arbitrary torsion-free groups, the argument above does not work a priori because the natural map from $M$ to $N / \equiv$ does not seem to be an order-embedding. A deeper reason for the embedding problem to be more difficult in the torsion-free case is that every divisible torsion-free commutative monoid is unperforated, while there are perforated torsion-free cones. But normal divisibility plays an important role in the proof of Corollary 2.7, even for the part about refinement! However, we will see in next chapter that the result of Corollary 2.9 still holds for torsion-free partially ordered abelian groups (and even more general classes of commutative monoids) as far as refinement is concerned.

Finally, let us prove the "simple analogue" of Proposition 1.16:

Proposition 2.12. $\quad$ Let $\mathbf{C}$ be the class of all antisymmetric cones. Then every simply $\mathbf{C}$-closed cone satisfies (WSD).

Therefore, every simple antisymmetric cone order-embeds into a simple normally divisible refinement cone satisfying (WSD).

Proof. Let $M$ be a simply C-closed cone. By Proposition 1.5, $M$ orderembeds into a C-closed cone $N$. By Proposition 1.16, $N$ satisfies (WSD), while by Theorem 2.6, $M$ is a normally divisible refinement cone. Now let $a_{0}, a_{1}, b$ and $c$ be elements of $M$ such that $a_{0}+a_{1}+c=b+c$. Consider the following equation system with unknowns $\mathbf{x}_{0}$ and $\mathbf{x}_{1}$ :

$$
\Sigma:\left\{\begin{array}{l}
\mathbf{x}_{0}+c=a_{0}+c \\
\mathbf{x}_{1}+c=a_{1}+c \\
\mathbf{x}_{0}+\mathbf{x}_{1}=b
\end{array}\right.
$$

Our goal is to prove that $\Sigma$ admits a solution in $M$. One deals with the easy cases $a_{0}=0$ or $a_{1}=0$ or $b=0$ just as at the beginning of the proof of Proposition 1.16. 
Thus suppose from now on that $a_{0}, a_{1}$ and $b$ are all non zero. By Lemma 2.2 and Theorem 2.6, there exists $d \neq 0$ in $M$ such that $d \leq a_{i}($ all $i<2$ ) and $2 d \leq b$. Thus there are elements $a_{i}^{\prime}(i<2)$ and $b^{\prime}$ of $M$ such that $a_{i}=a_{i}^{\prime}+d$ (all $i<2$ ) and $b=b^{\prime}+2 d$. Therefore, $a_{0}^{\prime}+a_{1}^{\prime}+c+2 d=b^{\prime}+c+2 d$, whence, applying twice the normal divisibility of $M$, there are elements $u_{i}(i<2)$ of $M$ such that $2 u_{i}=d($ all $i<2)$ and $a_{0}^{\prime}+a_{1}^{\prime}+u_{0}+u_{1}+c=b^{\prime}+u_{0}+u_{1}+c$. Since $N$ satisfies (WSD), there are elements $y_{0}$ and $y_{1}$ of $N$ such that $a_{i}^{\prime}+u_{i}+c=y_{i}+c$ (all $i<2$ ) and $b^{\prime}+u_{0}+u_{1}=y_{0}+y_{1}$. Put $x_{i}=y_{i}+u_{i}$. Then it is easy to verify that $\left(x_{0}, x_{1}\right)$ is a solution of $\Sigma$ in $N$. Furthermore, $u_{i} \leq x_{i} \leq a_{i}+c$ and $2 u_{i}=d$ with $d>0$ in $M$, thus, since $M$ is simple, $x_{i} \asymp d$. Thus $\Sigma$ admits a solution in $N(d)$ which is a simple (conical) extension of $M$, whence, by assumption on $M, \Sigma$ admits a solution in $M$.

\section{$\S 3$. The torsion-free case.}

As we have seen in Remark 2.11, the torsion-free case cannot be handled directly by the methods of previous sections, the main problem being divisibility. Thus we will need to make up for this by introducing a new condition, weaker than divisibility, which we will naturally call as in [13, definition 2.19] quasidivisibility.

Throughout this section, we will fix a multiplicative subsemigroup $\mathbb{P}$ of $\mathbb{N}$ such that $\mathbb{P} \nsubseteq\{1\}$.

Lemma 3.1. Every $\mathbb{P}$-torsion-free commutative monoid is separative.

Proof. Fix an element $p$ of $\mathbb{P} \backslash\{1\}$. If $M$ is $\mathbb{P}$-torsion-free, then for all $a, b \in M$ such that $2 a=a+b=2 b$, then an easy induction proof shows that $(k+l) a=k a+l b=(k+l) b$ for all $k, l \in \mathbb{N}$. In particular, $p a=p b$; whence $a=b$. Thus $M$ is separative.

Definition 3.2. A commutative monoid is quasi-divisible when it satisfies the following axiom:

$$
(\forall x)(\exists u, v)(2 u+3 v=x)
$$

Lemma 3.3. Every quasi-divisible commutative monoid satisfies the statement

$$
(\forall x)(\exists y)(2 y \leq x \leq 3 y) .
$$

Proof. Let $u, v$ such that $x=2 u+3 v$. Take $y=u+v$.

Lemma 3.4. Let $M$ be a separative quasi-divisible refinement cone, let $a \in M$. Then $M(a)$ is a simple, separative quasi-divisible refinement cone.

Proof. Simplicity has already been proved in 2.3. Separativity is trivial. Now let $b \in M(a)$, we will find solutions in $M(a)$ of the equation $2 u+3 v=b$. Since $M$ is quasi-divisible, three successive applications of Lemma 3.3 yield easily an element $c$ of $M$ such that $5 c \leq b$ and $c \asymp b$, so that $c \in M(a)$; let $b^{\prime} \in M$ such that $b=5 c+b^{\prime}$. Since $M$ is quasi-divisible, there exist $u^{\prime}$ and $v^{\prime}$ in $M$ such that $b^{\prime}=2 u^{\prime}+3 v^{\prime}$. Now note that $b=2 u+3 v$ where both $u=c+u^{\prime}$ and $v=c+v^{\prime}$ belong to $M(a)$; thus $M(a)$ is quasi-divisible. 
Let us check now that $M(a)$ is a refinement monoid. Thus let $a_{i}, b_{i}$ $(i<2)$ be elements of $M(a)$ such that $a_{0}+a_{1}=b_{0}+b_{1}$; consider as usual the following equation system

$$
\Sigma: \begin{cases}\mathbf{x}_{i 0}+\mathbf{x}_{i 1}=a_{i} & (\text { all } i<2) \\ \mathbf{x}_{0 i}+\mathbf{x}_{1 i}=b_{i} & (\text { all } i<2)\end{cases}
$$

If $a_{i}=0$ or $b_{i}=0$ for some $i$, then it is obvious that $\Sigma$ admits a solution in $M(a)$. Thus suppose that all the $a_{i}, b_{i}$ 's are non zero. Thus $a_{0} \asymp a_{1} \asymp b_{0} \asymp b_{1}$. Let $X=\left\{a_{0}, a_{1}, b_{0}, b_{1}\right\}$. By Lemma 2.2, there exists $c \asymp a$ such that $c \leq X$. Since $M$ is quasi-divisible and by two successive applications of Lemma 3.3, there exists $d \in M$ such that $3 d \leq c$ and $d \asymp c$. Thus there exist elements $a_{i}^{\prime}, b_{i}^{\prime}(i<2)$ of $M$ such that $a_{i}=3 \bar{d}+a_{i}^{\prime}$ and $b_{i}=3 d+b_{i}^{\prime}$. Hence $a_{0}^{\prime}+a_{1}^{\prime}+6 d=b_{0}^{\prime}+b_{1}^{\prime}+6 d$. Now, we use the separativity of $M$ (see Lemma 3.1): this allows us to infer that $a_{0}^{\prime}+a_{1}^{\prime}+d=b_{0}^{\prime}+b_{1}^{\prime}+d$. Therefore, one can form a refinement matrix with entries in $M$ as follows:

\begin{tabular}{|c|c|c|}
\cline { 2 - 3 } \multicolumn{1}{c|}{} & $b_{0}^{\prime}+d$ & $b_{1}^{\prime}$ \\
\hline$a_{0}^{\prime}+d$ & $p$ & $q$ \\
\hline$a_{1}^{\prime}$ & $r$ & $s$ \\
\hline
\end{tabular}

Thus the following is also a refinement matrix:

\begin{tabular}{|c|c|c|}
\cline { 2 - 3 } \multicolumn{1}{c|}{} & $b_{0}$ & $b_{1}$ \\
\hline$a_{0}$ & $p+d$ & $q+d$ \\
\hline$a_{1}$ & $r+d$ & $s+2 d$ \\
\hline
\end{tabular}

and all its entries belong to $M(a)$; whence $M(a)$ is a refinement monoid.

Now we come to the main theorem of this section.

Theorem 3.5. Let $M$ be $\mathbf{C}_{\mathbb{P}}$-strongly unitarily closed. Then $M$ is a quasidivisible refinement cone.

Recall (Lemma 3.1) that $M$ is also separative.

Proof. We shall use throughout this proof the techniques of amalgamated sums used in section 1 .

Let us prove quasi-divisibility. Thus let $a \in M$; consider the equation $\Sigma: 2 \mathbf{x}+3 \mathbf{y}=a$. If $a=0$, then $\Sigma$ trivially admits a solution in $M$. Thus suppose that $a \neq 0$. Let $D$ be the subgroup of $\mathbb{Z}^{2}$ generated by $(2,3)$, let $e: D^{+} \rightarrow$ $\left(\mathbb{Z}^{+}\right)^{2}$ be the inclusion map. Let $f: D^{+} \rightarrow M$ be the unique homomorphism such that $f((2,3))=a$; note that $f$ is conical. Let $N=\left(\mathbb{Z}^{+}\right)^{2} \amalg_{e, f} M$, let $\bar{e}: M \rightarrow N$ and $\bar{f}:\left(\mathbb{Z}^{+}\right)^{2} \rightarrow N$ be the natural homomorphisms. Since the map $n \mapsto(n, n)+D$ is easily seen to be an isomorphism from $\mathbb{Z}$ onto $\mathbb{Z}^{2} / D$, the latter is torsion-free; it follows easily that $e$ is strongly unitary. By Lemma 1.6, $N$ is conical and $\bar{e}$ is strongly unitary.

Now let $\sim$ be the congruence defined on $N$ by

$$
x \sim y \Leftrightarrow(\exists p \in \mathbb{P})(p x=p y) .
$$

It is obvious that $N / \sim$ is conical. By construction, $N / \sim$ is $\mathbb{P}$-torsion-free. Let $\pi: N \rightarrow N / \sim$ be the natural projection and let $j=\left.\pi\right|_{M}$. Since $M$ is $\mathbb{P}$-torsionfree, $j$ is one-to-one. Since $M$ is cofinal in $N, j$ has cofinal image. Let $x, y \in M$ 
and $z \in N$ such that $j(x)+\pi(z)=j(y)$. This means that there exists $p \in \mathbb{P}$ such that $p(x+z)=p y$, i.e., $p x+p z=p y$. Since $N$ is a unitary extension of $M$, it follows that $p z \in M$. Therefore, by strong unitarity, $z \in M$, whence $\pi(z) \in j[M]$ : hence, $j$ is unitary. Finally, let $z \in N$ and $m \in \mathbb{N}$ such that $m \pi(y) \in j[M]$. This means that there exists $x \in M$ such that $m y \sim x$, thus there exists $p \in \mathbb{P}$ such that $p m y=p x$; thus $p m y \in M$, thus, since $e$ is strongly unitary, $y \in M$, whence $\pi(y) \in j[M]$. Therefore, $j$ is strongly unitary. Thus identify $M$ with $j[M]$.

But the equation $\Sigma$ obviously admits a solution in $N$ (viz. $\quad(x=$ $[(1,0), 0] ; y=[(0,1), 0])$ with the notations of Lemma 1.6), thus also in $N / \sim$. Since $N / \sim$ is a $\mathbb{P}$-torsion-free strong unitary (conical) extension of $M$ and by assumption on $M$, the equation also admits a solution in $M$. Hence we have checked quasi-divisibility of $M$.

Finally, the fact that $M$ is a refinement cone results from Theorem 1.14 (a).

Corollary 3.6. $\quad$ Every $\mathbb{P}$-torsion-free cone embeds strongly unitarily into a $\mathbb{P}$-torsion-free quasi-divisible refinement cone.

Again, the proof of Corollary 1.12 allows us to extend this result to other classes:

Corollary 3.7. $\quad$ Every stably finite $\mathbb{P}$-torsion-free cone embeds strongly unitarily into a stably finite $\mathbb{P}$-torsion-free quasi-divisible refinement cone.

Corollary 3.8. $\quad$ Every directed $\mathbb{P}$-torsion-free partially ordered abelian group $A$ embeds cofinally into a directed $\mathbb{P}$-torsion-free Riesz group $B$ with quasi-divisible positive cone, with in addition $B / A$ torsion-free.

Proof. Let $\mathbf{C}$ be the class of all cancellative $\mathbb{P}$-torsion-free cones. It suffices to prove that every $\mathbf{C}$-strongly unitarily closed element $M$ of $\mathbf{C}$ is a quasi-divisible refinement cone. By Corollary 3.6, $M$ embeds strongly unitarily into a $\mathbb{P}$-torsionfree quasi-divisible refinement cone $N$. Now define a congruence $\equiv$ on $N$ by putting

$$
x \equiv y \Leftrightarrow(\exists z)(x+z=y+z) .
$$

As in the proof of the Claim of Theorem 1.9, one proves that $N / \equiv$ is conical. By using the fact that $M$ is strongly unitary (in particular, cofinal) in $N$, it is easy to verify that the natural homomorphism $j: M \rightarrow N / \equiv$ is strongly unitary. Now identify $M$ and $j[M]$. By using the fact that $N$ is $\mathbb{P}$-torsion-free, it is also easy to verify that $N / \equiv$ is still $\mathbb{P}$-torsion-free. Thus $N / \equiv$ belongs to C.

The rest of the proof is automatic: let for example $a \in M$. Since $N$ is quasi-divisible, the equation $2 \mathbf{x}+3 \mathbf{y}=a$ admits a solution in $N$, thus also in $N / \equiv$. Since the latter is a strong unitary extension of $M$ and by assumption on $M$, the equation also admits a solution in $M$. The proof for refinement is similar.

Let us now turn our attention on simple $\mathbb{P}$-torsion-free cones. The "simple analogue" of Theorem 3.5 is the following: 
Theorem 3.9. $\quad$ Let $M$ be simply $\mathbf{C}_{\mathbb{P}}$-strongly unitarily closed. Then $M$ is a quasi-divisible refinement cone.

Proof. By Corollary 3.6, $M$ embeds strongly unitarily into some $\mathbb{P}$-torsionfree quasi-divisible refinement cone $N$.

Let us first prove quasi-divisibility of $M$. Thus let $a \in M$, we solve in $M$ the equation $\Sigma: 2 \mathbf{x}+3 \mathbf{y}=a$. If $a=0$ then there is trivially a solution, so suppose that $a \neq 0$. Since $N(a)$ is quasi-divisible (Lemma 3.4), $\Sigma$ admits a solution in $N(a)$. Since $a \neq 0$ and $M$ is simple, $M \subseteq N(a)$, thus $N(a)$ is a strong unitary extension of $M$, and since $N(a)$ is simple and belongs to $\mathbf{C}_{\mathbb{P}}, \Sigma$ admits a solution in $M$. Therefore, $M$ is quasi-divisible.

Let us check now that $M$ is a refinement monoid. Thus let $a_{i}, b_{i}(i<2)$ be elements of $M$ such that $a_{0}+a_{1}=b_{0}+b_{1}$; consider as usual the following equation system

$$
\Sigma^{\prime}: \begin{cases}\mathbf{x}_{i 0}+\mathbf{x}_{i 1}=a_{i} & (\text { all } i<2) \\ \mathbf{x}_{0 i}+\mathbf{x}_{1 i}=b_{i} & (\text { all } i<2)\end{cases}
$$

If $a_{i}=0$ or $b_{i}=0$ for some $i$, then it is obvious that $\Sigma$ admits a solution in $M$. Thus suppose that all the $a_{i}, b_{i}$ 's are non zero. Then, since $M$ is simple, all four of them are order-units, thus $a_{0} \asymp a_{1} \asymp b_{0} \asymp b_{1}$. By Lemma 3.4, $N\left(a_{0}\right)$ is a refinement cone, thus $\Sigma^{\prime}$ admits a solution in $N\left(a_{0}\right)$. Thus as before, $\Sigma^{\prime}$ admits a solution in $M$, and $M$ is a refinement cone.

Corollary 3.10. Every simple $\mathbb{P}$-torsion-free cone embeds strongly unitarily into a simple $\mathbb{P}$-torsion-free quasi-divisible refinement cone.

Furthermore, as for Corollary 3.7, one obtains easily

Corollary 3.11. Every simple $\mathbb{P}$-torsion-free stably finite cone embeds strongly unitarily into a simple $\mathbb{P}$-torsion-free stably finite quasi-divisible refinement cone. following:

Now, a proof similar to the proof of Corollary 3.8 yields easily the

Corollary 3.12. $\quad$ Every simple $\mathbb{P}$-torsion-free partially ordered abelian group $A$ embeds cofinally into a simple $\mathbb{P}$-torsion-free Riesz group with quasi-divisible positive cone $B$ such that in addition, $B / A$ is torsion-free.

Remark 3.13. In fact, a direct (though not devoid of lengthy calculations...) construction shows that every partially ordered abelian group (resp. simple partially ordered abelian group) $G$ embeds cofinally into an interpolation group (resp. simple Riesz group) $H$ such that in addition, if $F$ is the free abelian group with $\left|G^{+}\right|$generators, then $H=G \oplus F$ as abelian groups. Note that this implies immediately that if $G$ is torsion-free, then so is $H$.

Corollary 3.12 also allows us to construct the following example (the question which it answers was communicated to us by K. R. Goodearl):

Example 3.14. A countable torsion-free simple Riesz group $G$ and an interval (i.e., a nonempty, upward directed lower subset of $G^{+}$) $D$ of $G^{+}$such that $2 D=G^{+}$but $D \neq G^{+}$.

Proof. We shall first construct an example with all the properties above except interpolation; then we will conclude by Corollary 3.12. 
is,

To start with, let $A$ be the submonoid of $\mathbb{Z}^{+}$generated by $\{2,7\}$ : that

$$
A=\{0,2,4,6,7,8,9,10,11, \ldots\}
$$

and let $M$ be the submonoid of $\mathbb{Q}^{+}$generated by all elements of the form $(k / 2)(9 / 2)^{n}$ where $k \in A$ and $n \in \mathbb{Z}^{+}$. Let $G_{0}=M+(-M)$, equipped with the positive cone $M$. Since $G_{0}$ is directed and $G_{0}^{+}=M$ is a submonoid of $\mathbb{Q}^{+}, G_{0}$ is a simple partially ordered abelian group. For all $n \in \mathbb{Z}^{+}$, put $d_{n}=(9 / 2)^{n}$. Note that $d_{n}=(2 / 2)(9 / 2)^{n}, d_{n+1}-d_{n}=(7 / 2)(9 / 2)^{n}$ and $2 d_{n+1}-4 d_{n}=(10 / 2)(9 / 2)^{n}$, whence the following claim:

Claim 1. For all $n \in \mathbb{Z}^{+}$, all elements $d_{n}, d_{n+1}-d_{n}$ and $2 d_{n+1}-4 d_{n}$ belong to $M$.

Since, by Claim 1 , the sequence $\left(d_{n}\right)_{n}$ is increasing in $G_{0}$, it generates an interval $D_{0}$, viz.

$$
D_{0}=\left\{x \in G_{0}^{+}:\left(\exists n \in \mathbb{Z}^{+}\right)\left(x \leq_{G_{0}} d_{n}\right)\right\}
$$

Claim 2. $2 D_{0}=G_{0}^{+}$.

Proof of Claim. It is easy to see that every element of $G_{0}$ is bounded above (for $\left.\leq_{G_{0}}\right)$ by some $m\left(d_{0}+d_{1}+\cdots+d_{n}\right)$ where $m, n \in \mathbb{N}$, thus by $m n d_{n}$, thus by some $2^{k} d_{n}(k \in \mathbb{N})$. But an easy induction proof (using Claim 1) shows that $2^{k} d_{n} \leq_{G_{0}} 2 d_{n+k-1} ;$ since $2 d_{n+k-1} \in 2 D_{0}$, the conclusion follows.

We shall now prove that $2 d_{0} \notin D_{0}$ (whence $D_{0} \neq G_{0}^{+}$). Towards this goal, we shall prove by induction on $m$ that $2 d_{0} \overleftarrow{L}_{G_{0}} d_{m}$ (all $m \in \mathbb{Z}^{+}$). It is trivial for $m=0$. Suppose that $m>0$ and that the result has been proved for all $m^{\prime}<m$, and suppose that the conclusion fails for $m$, i.e., there are $n \in \mathbb{Z}^{+}$ and $k_{l}(l \leq n)$ in $A$ such that

$$
(9 / 2)^{m}-2=\sum_{l \leq n}\left(k_{l} / 2\right)(9 / 2)^{l}
$$

Taking the minimal possible value for $n$ ensures that $k_{n} \neq 0$ (because $(9 / 2)^{m} \neq$ $2)$. Then $(9 / 2)^{m}-2 \geq(2 / 2)(9 / 2)^{n}$, whence $n<m$. On the other hand, the right-hand side of $(*)$ belongs to $2^{-n-1} \mathbb{Z}^{+}$, thus $2^{n+1}\left((9 / 2)^{m}-2\right) \in \mathbb{Z}^{+}$, thus $n+1 \geq m$. It follows that $m=n+1$. If $k_{n}$ were even, then the right-hand side of $(*)$ would belong to $2^{-n} \mathbb{Z}^{+}$, whence $2^{n}\left((9 / 2)^{n+1}-2\right) \in \mathbb{Z}^{+}$, a contradiction: thus $k_{n}$ is odd. If $k_{n} \geq 9$, then the right-hand side of $(*)$ would be $\geq(9 / 2)^{n+1}$, thus $>(9 / 2)^{m}-2$, a contradiction: thus the only possibility left is $k_{n}=7$, so that $\left(k_{n} / 2\right)(9 / 2)^{n}=(9 / 2)^{m}-(9 / 2)^{n}$. Hence, after canceling $(9 / 2)^{m}$ from $(*)$, we obtain

$$
(9 / 2)^{n}-2=\sum_{l<n}\left(k_{l} / 2\right)(9 / 2)^{l},
$$

with $n<m$, which contradicts the induction hypothesis. Thus we have proved that $2 d_{0} \notin D_{0}$.

By Corollary $3.12, G_{0}$ embeds cofinally into a torsion-free simple Riesz group $G$; a standard Löwenheim-Skolem type argument shows easily that one can take $G$ countable. If $D$ is the interval of $G^{+}$generated by $D_{0}$, it is then easy to see that $2 D=G^{+}$but $D \neq G^{+}$. 
Problem 3.15. Can one realize Example 3.14 as a torsion-free Riesz group of rank one (i.e., with positive cone an additive submonoid of $\mathbb{Q}^{+}$)? The latter were for example studied in [10].

Final comments. As mentioned in the Introduction, we chose here to restrict ourselves essentially to conical commutative monoids and all our results here can be extended, modulo sometimes minor changes in the statements and the proofs, to arbitrary commutative monoids (for example, Lemma 1.6 (a) is no longer useful but some extra care has to be taken about the maximal subgroup of our monoids). The definition of $M(a)$ has then to be changed into $M(a)=\{x \in M$ : $x \asymp a$ or $x \leq 0\}$. Some results whose statements extend mutatis mutandis are for example 1.8, 1.9, 1.10, 1.12, 1.14, 1.15 (a)(c), 2.6, 2.7, 2.8, 2.10, 3.5 - 3.12. The statements of $1.16,1.17,2.12$ also extend to all commutative monoids, but antisymmetry is no longer needed among the hypotheses.

Acknowledgment. I wish to thank Ken Goodearl for his many helpful comments and corrections about this paper, and in particular for having pointed several oversights and a significant error in its first version.

\section{References}

[1] Bergman, G. M., unpublished correspondence (December 1990).

[2] Clifford, A. H., and G. B. Preston, "The algebraic theory of semigroups," Mathematical Surveys 7, American Mathematical Society, Providence, R.I., vol. 1, 1961 and vol. 2, 1967.

[3] Dobbertin, H., Refinement monoids, Vaught Monoids, and Boolean Algebras, Mathematische Annalen 265 (1983), 475-487.

[4] Goodearl, K. R., "Von Neumann Regular Rings", Pitman, London, 1979.

[5] Goodearl, K. R., "Partially ordered abelian groups with the interpolation property", Mathematical surveys and monographs, number 20, American Mathematical Society, 1986.

[6] Goodearl, K. R., Von Neumann regular rings and direct sum decomposition problems, Abelian Groups and Modules, Padova 1994 (A. Facchini and C. Menini, eds.), Dordrecht (1995) Kluwer, 249-255.

[7] Grillet, P. A., Interpolation properties and tensor products of semigroups, Semigroup Forum 1 (1970), pp. 162-168.

[8] Howie, J. M., "An introduction to semigroup theory", Academic Press: London, New York, San Francisco, 1976.

[9] Moncasi, J., A regular ring whose $K_{0}$ is not a Riesz group, Communications in Algebra 13 (1985), 125-133.

[10] Pardo, E., On the representation of simple Riesz groups, to appear in Communications in Algebra.

[11] Wehrung, F., Injective positively ordered monoids I, Journal of Pure and Applied Algebra 83 (1992), 43-82.

[12] Wehrung, F., Restricted injectivity, transfer property and decompositions of separative positively ordered monoids, Communications in Algebra 22 (5) (1994), 1747-1781.

[13] Wehrung, F., Monoids of intervals of ordered abelian groups, Journal of Algebra 182 (1996), pp. 287-328.

[14] Wehrung, F., Tensor products of structures with interpolation, Pacific Journal of Mathematics 176 (1) (1996), 267-285. 
Université de Caen

Département de Mathématiques 14032 Caen Cedex

FRANCE

e-mail: gremlin@math.unicaen.fr 